\title{
Steganography Based Human Skin Using Wavelet Transformation in RGB Image
}

\author{
${ }^{1}$ Jamila Harbi S., ${ }^{2}$ Zeyad Nabeel N. \\ ${ }^{1}$ AL-Mustansiriyah University, College of Science, Dept. Computer Sciences \\ ${ }^{2}$ Karbala University, College of Science, Dept. Physics \\ E-mail address: dr.jameelahharbi@yahoo.com; ziyad.nabeel@gmail.com
}

Keywords: steganography Based Human Skin Using; Wavelet Transformation; Image

\begin{abstract}
Steganography is the art and science that enables you to hide the data in certain communication such as (video, Audio, and image). Here it doesn't scramble the data and send it, but we hide it using a color image to hide the data at the Human skin color and doing that using the cropping phrase to get the required area to hide data. In this paper, we will use the Wavelet and slant, let to process the hiding in the blue band of the RGB image and apply the DWT (discrete wavelet transformation) to get the decomposed bounds of the Cover image to use it to hide the data of the (stage) image.
\end{abstract}

\section{INTRODUCTION}

Steganography is the art and the science of hiding any kind of data in certain communication media such (video, audio, image) [1]. Due to the great increase of the internet usage in the recent arena, there were greater needs to secure information that is transmitted through the World Wide Web [2]. The hiding of the information is different from cryptography where the ferries are a method to embed the original data without having them changed in a certain a medium while the cryptography is changing the data that is hidden in other model till it can't be understood [3]. Using the skin tone color is one of the most important ways to do hiding where we use the skin and then use the blue or green component of the RGB image to embed the data into that bond and then recombine that main image and apply a certain feudality to find out that the stage image is the same as the original image. In this paper, we will use the Wavelet to transform the cover image into the frequency domain after we get the blue band and then using the slantlet on the stage image, after that we embed the data in the $\mathrm{HH}$ bond of the Wavelet and recombine the image, then we measure the change in the stage image and the original one.

\section{SKIN DETECTION}

Human skin is a good place to hide the data into that based on the experimental result where a cover image will be detected in skin in the RGB color system to get the skin first [3].

For that a special algorithm is made to get the suitable space of colors that works properly with the skin tone for different kinds of skin colors. Mainly there are too many color systems that work in that field that are called the biometric operation [3].

In here we will use the RGB color to do that skin detection directly without transforming the image (original image) into any other system to get the maximum use of the skin features and then use the Blue band to embed the data into where the blue color is the familiar to the human eye and the changing in it will not be recognized easily [4]. 


\section{CROPPING}

It is the process of getting a required area to work with it in the human skin tone and that enables the system to get rid of the un necessary tons of colors in any image as we will see in the result.

\section{Discrete Wavelet Transformation(DWT)}

It is a frequency domain transformation that works with steganography to prepare the image to be ready to embedding process, where it divides the image in to numerous frequency channels that are named as fellow:

LL- Horizontal and vertical low pass filter.

LH- Horizontal low pass and vertical high pass filter.

HL- Horizontal high pass and vertical low pass filter.

$\mathrm{HH}$ - Horizontal and vertical high pass filter.

Since the human eye is sensitive to low frequency, that we will use the high frequency domain to hide the secret data[3]. Embedding data can be done in one of the level of the decomposition of the DWT that made to the image, that will give the work the reliability and power to pass the attack from hackers or unwanted third person[5].

\section{Slantlet Wavelet}

The Slantlet Transform (SLT) is a recently developed multiresolution technique especially well-suited for piecewise linear data. SLT has proved that it is a better candidate for signal compression as compared to the conventional DCT and DWT based method and SLT based algorithms retain higher percentage of energy after compression compared to the DWT approach. Moreover, SLT has been used in medical image processing for the classification of magnetic resonance human brain images, also presented as based method for image finger prints [4].

The Slantlet filters are based on the parallel structure of DWT filter bank as shown in Figure (1). Slantlet is employing different filters for each scale whereas DWT is usually implemented in the form of an iterated filterbank, utilizing a tree structure. With this extra degree of freedom obtained by giving up the product form, filters of shorter length are designed satisfying orthogonality and zero moment conditions where $H(Z) \& F(Z)$ are filters of length of 4 [6].

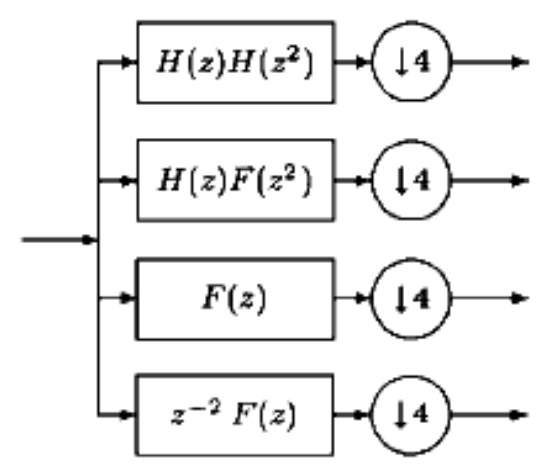

Fig 1. Tow -scale filterbank and an equivalent structure [5] 


\section{Proposed work}

1- Cover image algorithm

\section{2- secret image algorithm}
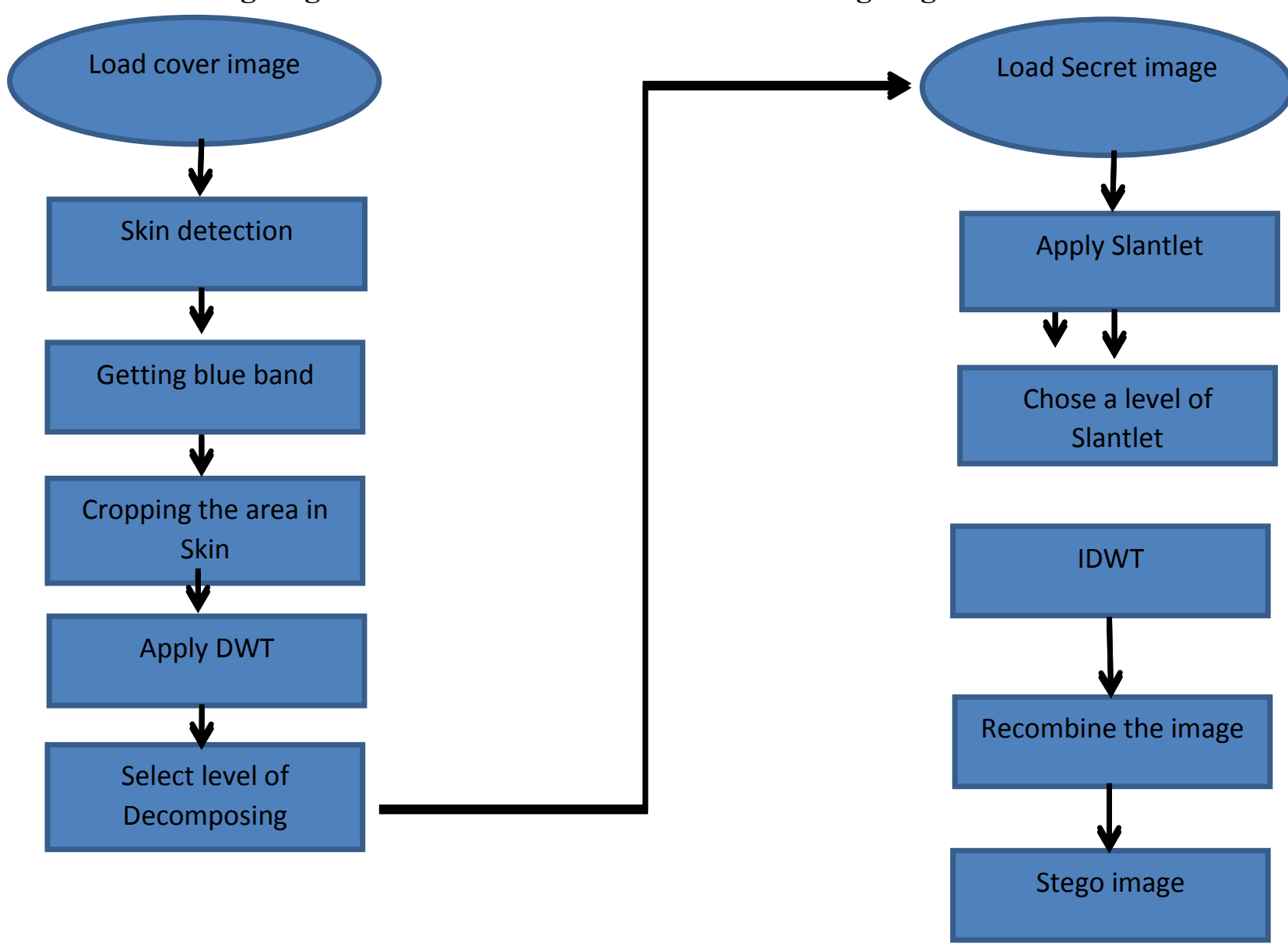

\section{Preparing Cover image:}

1- load the cover image in the form of RGB.

2- skin detection preformed on cover image.

3- Split the image into R,G,and Blue component to get the blue band.

4- Apply DWT on blue image.

5- Select level of Decompsion to hide the secrt image.

Preparing the Secret image:

1- Load Secret imge in gray level form.

2- Apply Slanlet Filter onit.

3- Chose a level of Slantlet.

4- Embed the data by applying IDWT

5- Recombine the image.

Recombine the origenal image to preform the fedality mesurement. 


\section{3- Exrxction Algorithm}

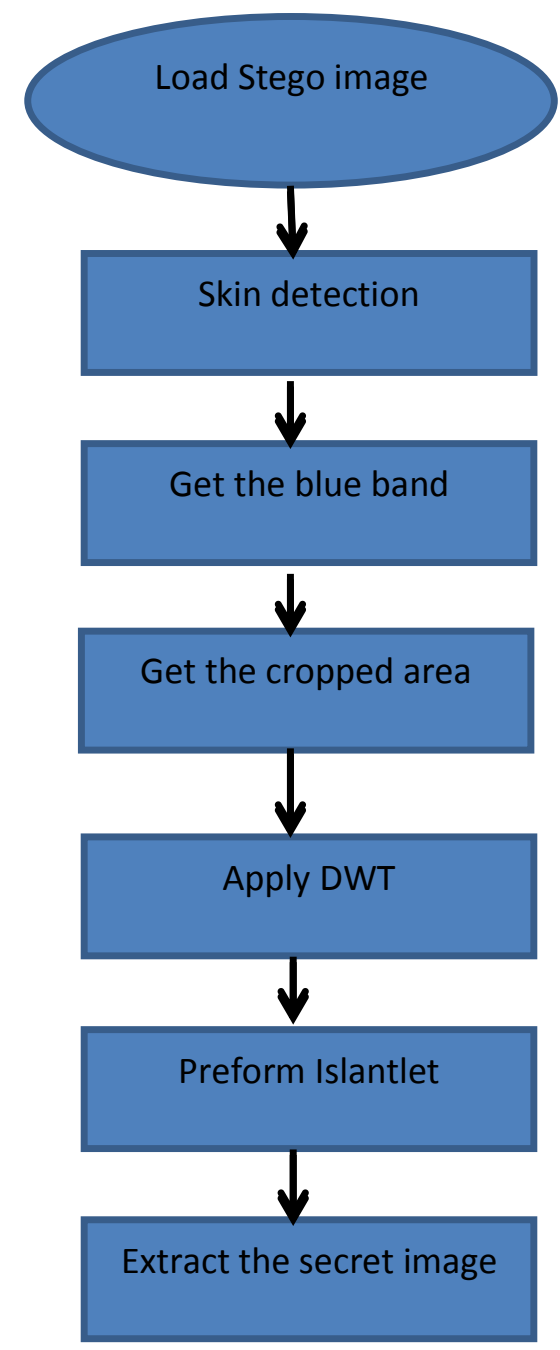

1- Load the Stego image.

2- Skin detection is to be performed.

3- Split it into the Red, Green, Blue bands to get the Blue band

4- Cropping the required part of the image.

5- Apply DWT to get the internal Bands.

6- Preform Islantlet.

7- Extracting the secret image.

\section{EXPERIMENTAL RESULTS} below:

By the use of the algorithem that showen in the above figures, we will get the resulted listed

\begin{tabular}{|c|c|c|c|}
\hline \multicolumn{2}{|c|}{$\begin{array}{l}\text { Data For White Skin and Different Area of } \\
\text { Cropping }\end{array}$} & & \\
\hline area of Cropping & MSE & PSNR & \\
\hline $15 \times 15$ & 0.06 & 74.65 & \multirow{3}{*}{ 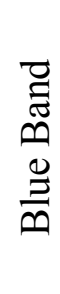 } \\
\hline $25 \times 25$ & 0.1 & 69.69 & \\
\hline $35 \times 35$ & 0.16 & 65.51 & \\
\hline
\end{tabular}




\begin{tabular}{|c|c|c|}
\hline $45 \times 45$ & 0.47 & 56.17 \\
\hline $55 \times 55$ & 0.59 & 54.03 \\
\hline
\end{tabular}

\begin{tabular}{|c|c|c|c|}
\hline \multicolumn{2}{|c|}{ Data Fo rsugar Skin } & & \\
\hline Area of Cropping & MSE & PSNR & \multirow{6}{*}{ 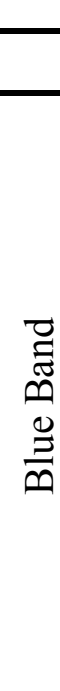 } \\
\hline $15 \times 15$ & 0.03 & 78.86 & \\
\hline $25 \times 25$ & 0.08 & 71.17 & \\
\hline $35 \times 35$ & 0.35 & 58.63 & \\
\hline $45 \times 45$ & 1.97 & 43.61 & \\
\hline $51 X 51$ & 2.94 & 40.14 & \\
\hline \multicolumn{2}{|c|}{ DATA For the Dark Skin } & & \multirow{7}{*}{ 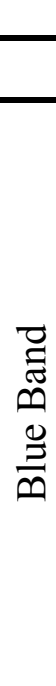 } \\
\hline Area of Cropping & MSE & PSNR & \\
\hline $15 \times 15$ & 0.11 & 68.49 & \\
\hline $25 \times 25$ & 0.27 & 60.96 & \\
\hline $35 \times 35$ & 0.55 & 50.63 & \\
\hline $45 X 45$ & 0.94 & 50.03 & \\
\hline $55 \times 55$ & 1.92 & 43.84 & \\
\hline
\end{tabular}

\section{CONCLUSION}

Applying the algorithim on different kinds of imags that have skin will give different result for each kind according to the skin color (While, Suger, Dark).

The tables showen above gives us the best way to steganography image using other image nd the correct skin color and the correct cropped image size to do the steganography, and to make sure that the data will be exract in correct way with not damging the original image. 


\section{Refrences}

[1] Biometric Steganographic Technique Using DWT and Encryption, Amritha.G. Meethu Varkey International Journal of Advanced Research in Computer Science and Software Engineering, Volume 3, Issue 3, March 2013 ISSN: 2277 128X

[2] A. Cheddad, J. Condell, K. Curran and P. Mc Kevitt, -Biometric inspired digital image Steganographyll, in: Proceedings of the 15th Annual IEEE International Conference and Workshops on the Engg.of Computer-Based Systems (ECBS,,08), Belfast, 2008, pp. 159168.

[3] ANALYSING SECURE IMAGE SECRET SHARING SCHEMES BASED ON STEGANOGRAPHY Sonali Patil, Kapil Tajane, Janhavi Sirdeshpande, INTERNATIONAL JOURNAL OF COMPUTER ENGINEERING \& TECHNOLOGY (IJCET) Volume 4, Issue 2, March - April (2013), pp. 172-178

[4] A. Cheddad, J. Condell, K. Curran and P. Mc Kevitt, "Biometric inspired digital image Steganography", in: Proceedings of the 15th Annual IEEE International Conference and Workshops on the Engineering of Computer-Based Systems (ECBS ${ }^{\text {ee }}$ 8), Belfast, 2008,

[5] Chang, C. C., Chen, T.S and Chung, L. Z., "A steganographic method based upon JPEG and quantization table modification," Information Sciences, vol.[4], pp. 123-138(2002).

[6] The Slantlet Transform Ivan W. Selesnick, Member, , IEEE TRANSACTIONS ON SIGNAL PROCESSING, VOL. 47, NO. 5, MAY 1999. 